\title{
Comprehensive Evaluation of AODV, DSR, GRP, OLSR and TORA Routing Protocols with varying number of nodes and traffic applications over MANETs
}

\author{
Gagangeet Singh Aujla ${ }^{1}$, Sandeep Singh Kang ${ }^{2}$ \\ ${ }^{1}$ (Department of C S E, Chandigarh Engineering College, India) \\ ${ }^{2}$ (Department of CS E, Chandigarh Engineering College, India)
}

\begin{abstract}
Wireless Technology is at its peak when we talk about research and innovation. This field has become a hub of invention of new theories and structures. Mobile Ad-hoc Network is a special point of focus for the researchers. MANET is a collection of wireless mobile nodes which forms a dynamic temporary network without using any existing infrastructure. In recent time the market of mobile devices, laptops, hand held and portable devices is at its zenith so communication between such devices is an important issue. Routing is an essential part in the success of communication among these mobile structures. Routing protocols play an important role for finding an efficient and reliable route from source to destination. In the literature, there are numerous MANET routing protocols aiming to find the most suitable path from source to destination. In this paper, a comprehensive simulation based performance study and analysis is performed on various types of routing protocols over MANET. Ad Hoc On-Demand Distance Vector (AODV), Dynamic Source Routing (DSR), Temporally-Ordered Routing Algorithm (TORA), Optimized Link State Routing (OLSR) and Geographic Routing Protocol (GRP) has been considered for investigation in this paper based on OPNET simulation. Moreover the performance of these routing protocols will be measured on the basis of throughput, delay, load and data dropped metrics.
\end{abstract}

Keywords - AODV, DSR, GRP, OLSR, TORA

\section{INTRODUCTION}

Network structure is changing rapidly in recent years. The only network available four decades ago was wired network. The emergence of wireless networks has gone a long way in solving the growing service demands. The focus of research and development has almost shifted from wired networks to wireless networks. The limitations of wireless network techniques such as high error rate, power restrictions, bandwidth constraints, etc has not deterred the growth of wireless networks. [1]. Mobile Ad-hoc network (MANET) is the most demanding field in the area of wireless networks. MANET has mobile devices or users which are generally known as nodes each one of which is equipped with radio transmitter and receiver [2]. MANET is a temporary network of wireless mobile nodes which has no fixed infrastructure. There are no dedicated routers, servers, access points, base stations and cables. The mobile nodes which are within each other's transmission range can communicate with each other directly otherwise, the nodes in between them forward the packets for them from source to destination. Every node acts as a router to forward the packets to other nodes whenever required [3].

One of the main areas of research has been routing technology which will route packets from source to destination. In this paper, we have evaluated performance of AODV, DSR, TORA, OLSR and GRP routing protocols based on e-mail and video conferencing traffic generating applications by increasing the number of nodes. The performance is analyzed by means of throughput, delay, load and data dropped by using OPNET Modeler 14.5. The rest of paper is organized as follows: Section II presents brief overview of MANET routing protocols that we evaluate. Section III describes simulation environment. Simulation results are shown in Section IV. Result analysis is done in Section V and finally, conclusion is drawn in section VI.

\section{MANET ROUTING PROTOCOLS}

There are several protocols proposed for wireless mobile ad-hoc networks. The first three protocols are selected from Reactive category namely AODV, DSR and TORA and the fourth is selected from proactive category namely OLSR whereas the last protocol is selected from Geographic Position Information based routing namely GRP.

\subsection{Ad-hoc On Demand Distance Vector (AODV)}

AODV is reactive routing protocol which does not discover or maintain a route until or unless requested by nodes. AODV uses destination sequence number to ensure the loop freedom and freshness of route [4]. AODV is capable of both unicast and multicast routing. The operation of protocols is divided into two 
functions: route discovery and route maintenance. When a node requests to communicate with another node it starts route discovery mechanism. The source node sends a route request message RREQ to its neighbors and if all those neighbor nodes do have any information about the destination node then they will further send the message to its neighbors and so on until the destination node is node is found. The node which has information of the destination node sends a route reply message RREP to the initiator of the RREQ message. The path is recorded in the intermediate nodes in the routing table and this path identifies the route. When the initiator receives the route reply message the route is ready and the initiator can start sending the packets. The route error RRER is reported when the link with the next hop breaks [5].

\subsection{Dynamic Source Routing (DSR)}

DSR is also a reactive routing protocol which uses the concept of source routing. In source routing the sender knows complete hop-by-hop route to the destination. All the routes are stored in the route cache. When a node attempts to send a data packet to a destination for which it does not know the route [6]. In DSR each node maintains a route cache with route entries which are continuously updated as and when route learns new routes [7]. The biggest advantage of DRS is that no periodic routing packets are required. DSR has also the capability to handle unidirectional links [8]. Unlike other protocols DSR requires no periodic packets of any kind at any layer within the network. The sender of the packets selects and controls the route used for its own packets, which also supports features such as load balancing. All routes used are guaranteed to be free of loops as the sender can avoid duplicate hops in the selected routes [9].

\subsection{Temporally Ordered Routing Algorithm (TORA)}

TORA is an adaptive on demand routing protocol for multi hop networks. TORA is source initiated specially proposed routing protocol for highly dynamic mobile, multi-hop wireless networks [8]. TORA is based on link reversal algorithms. TORA establish the routes quickly and minimize the communication overhead by localizing algorithm reaction to topological changes when possible [10]. Instead of using the concept of shortest path for computing routes which take huge amount of bandwidth TORA algorithm maintains the "direction of the next destination" to forward the packets. Thus the source node maintains one or two "downstream paths" to the destination node through multiple intermediate neighboring nodes. The three steps involved in TORA are: a) route creation, b) route maintenance, and c) route erasure. TORA uses the concept of "directed acyclic graphs" to establish downstream paths to destination and such DAG is known as "Destination Oriented DAG" [11].

\subsection{Optimized Link State Routing (OLSR)}

OLSR is a proactive routing protocol, in which all routes have route table for maintaining information to every node in the network. The routes are immediately available whenever needed due to the route tables. OLSR is an optimized version of link state protocol. OLSR uses the concept of Multipoint Relays (MPR) to reduce the possible overhead in the network. OLSR uses two types of control messages: Hello and Topology Control (TC). Hello message are used to find the link state and neighboring nodes. TC message is used to for broadcasting information for own advertized neighbors which includes at least the MPR selector list [12].

\subsection{Geographic Routing Protocol (GRP)}

GRP is a position based protocol classified as proactive routing protocol. In GRP the Global Positioning System (GPS) is used to mark the location of node and thee quadrants optimize flooding. When a node moves and crosses neighborhood then the flooding position is updated. The neighbors and their positions are identified by the exchange of "Hello" protocol. The concept of route locking ensures that a node can return its packet to the last node when it cannot keep on sending the packet to the next node. The network is divided into quadrants to reduce route flooding. The entire world is divided into quadrants from Lat, Long $(-90,-180)$ to Lat, Long $(+90,+180)$ [12]. Apart from actual geographic coordinates received by the GPS the other approach followed is Reference points in some fixed coordinate system [13].

\section{SIMULATION ENVIRONMENT}

The simulations are performed using OPNET Modeler 14.5 which supports the video conferencing and e-mail transmission. The nodes were spread randomly over an area of $1000 \mathrm{~m}$ x $1000 \mathrm{~m}$. The first scenario has 30 nodes, the second scenario has 60 nodes and the last scenario has 90 nodes. The simulation was run for 600 simulation seconds with a seed value of 128 . The mobility model used was "Random Waypoint Model". Random waypoint is most widely used mobility model in which a node randomly chooses a destination, called waypoint and moves towards it in a straight line with a constant velocity [13]. The simulation parameters used are listed in Table 1. 
Table 1: Simulation Parameters

\begin{tabular}{|c|c|}
\hline Simulation Parameter & Value \\
\hline Number of Nodes & 30,60 and 90 \\
\hline Simulation Time & $600 \mathrm{sec}$ (10 min) \\
\hline Simulation Area & $1000 \mathrm{~m}$ X 1000 m \\
\hline Routing Protocols & AODV, DSR, TORA, OLSR and GRP \\
\hline Node Movement Model & Random Waypoint \\
\hline Data Rate & $11 \mathrm{mbps}$ \\
\hline Application Name & $\begin{array}{c}\text { Video Conferencing (High resolution } \\
\text { video) and e-mail (High load) }\end{array}$ \\
\hline Bandwidth & 11 Mbps \\
\hline Simulator & Opnet Modeller 14.5 \\
\hline
\end{tabular}

The performance of the simulated results is analyzed according to different performance metrics. Such quantative measurement is useful as a prerequisite for assessing or evaluating the performance of network or even to compare the performance using different routing protocols. The following performance metrics are employed in this study:

- Throughput- Throughput is total packets successfully delivered to individual destination over total time divided by total time.

- Delay- It is the ratio of time difference between every packet sent and received to the total time difference over the total number of packets received.

- Load- Load represents the total load in bit/sec that all higher layers submit to wireless LAN layers in all WLAN nodes of the network.

- Data Dropped- Data dropped shows how many packets successfully sent and received across the whole network. It also explains the number of packet dropped during the transmission due to interference from other devices.

\section{Simulation RESULTS}

In this section we analyze the performance of routing protocol based on the results obtained after simulation experiments are conducted on routing protocols. The main target of this paper is to evaluate the performance and behavior of each routing protocol with respect to the effect of varying the number of nodes for two different applications i.e. video conferencing and e-mail. The results are based on evaluation metrics of delay, load, throughput and data dropped. We have divided our study into three sets of experiments: the first set studies the performance of five protocols over a small number of nodes (30 nodes) while the second and third consists of 60 and 90 nodes.

\subsection{Delay}

The Fig. 1(a) shows the entire delay for 30 nodes for email application. The GRP protocol shows least delay followed by OLSR and AODV. The TORA protocol shows highest delay. The Fig. 1(b) shows the entire delay for 30 nodes for video conferencing application. The GRP protocol shows least delay followed by TORA. The DSR protocol shows highest delay.

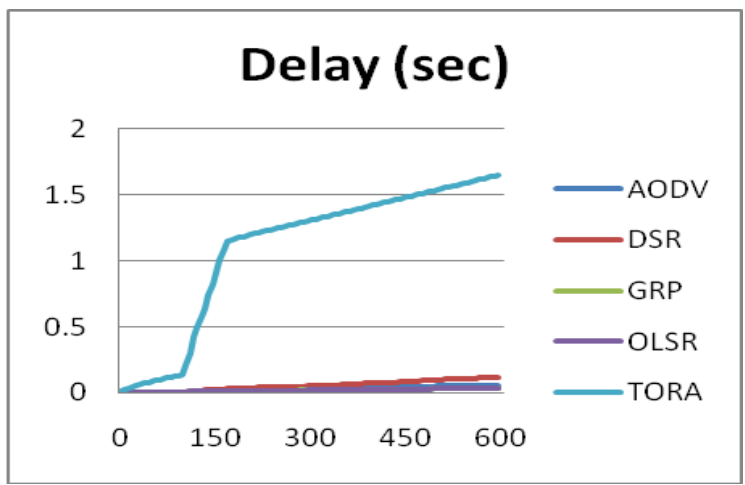

Fig. 1(a): Delay for 30 nodes for e-mail

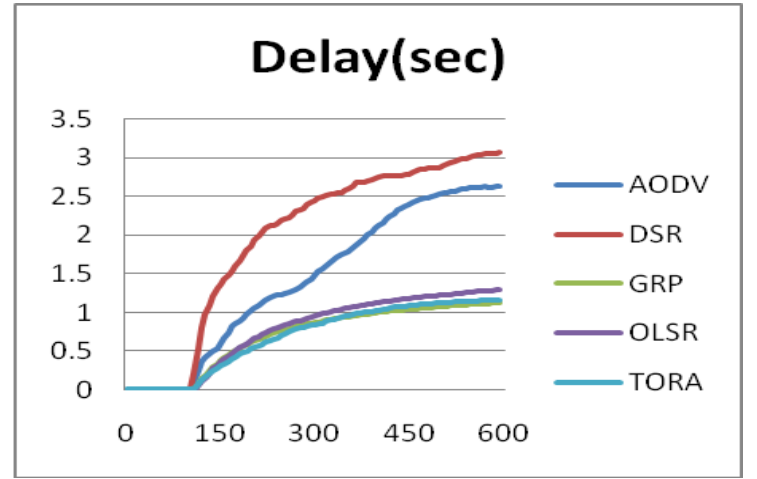

Fig. 1(b): Delay for 30 nodes for video conferencing 
The Fig. 2(a) shows the delay for 60 nodes for email application. The GRP shows least delay followed by OLSR. The TORA protocol shows highest delay. The Fig. 2(b) shows the delay for 60 nodes for video conferencing. The GRP shows least delay followed by OLSR. The TORA protocol shows highest delay.

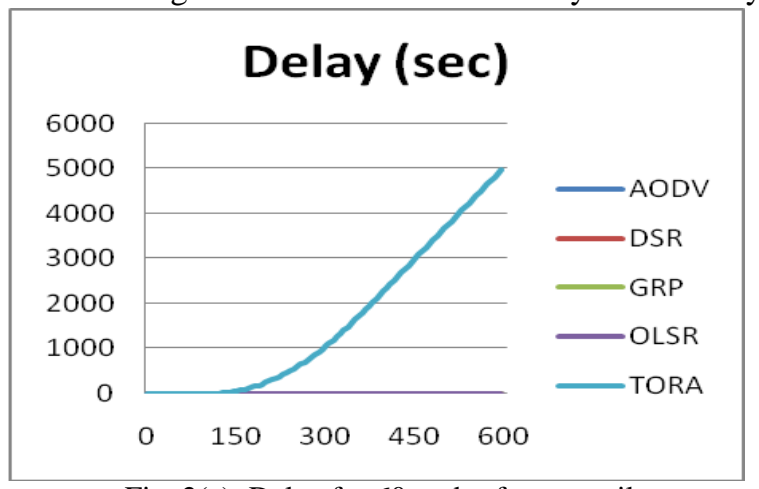

Fig. 2(a): Delay for 60 nodes for e-mail

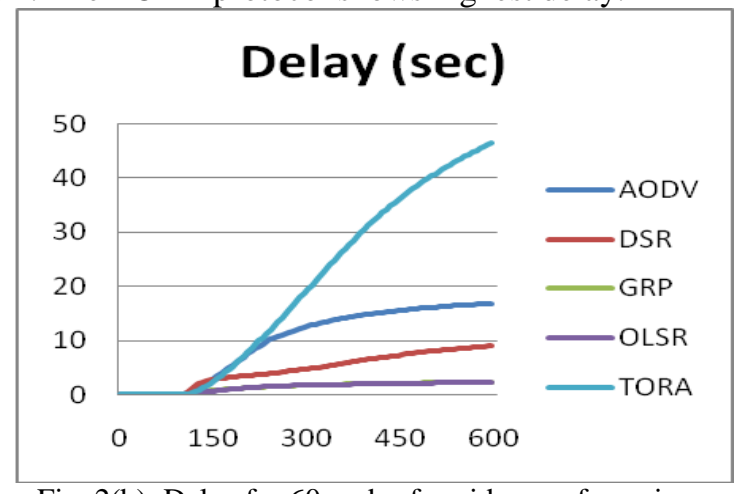

Fig. 2(b): Delay for 60 nodes for video conferencing

The Fig. 3(a) shows the entire delay for 90 nodes for email. The GRP shows least delay followed by OLSR. The TORA protocol shows highest delay. The Fig. 3(b) shows the entire delay for 90 nodes for video conferencing application. The OLSR shows least delay followed by GRP. The TORA protocol shows highest delay.

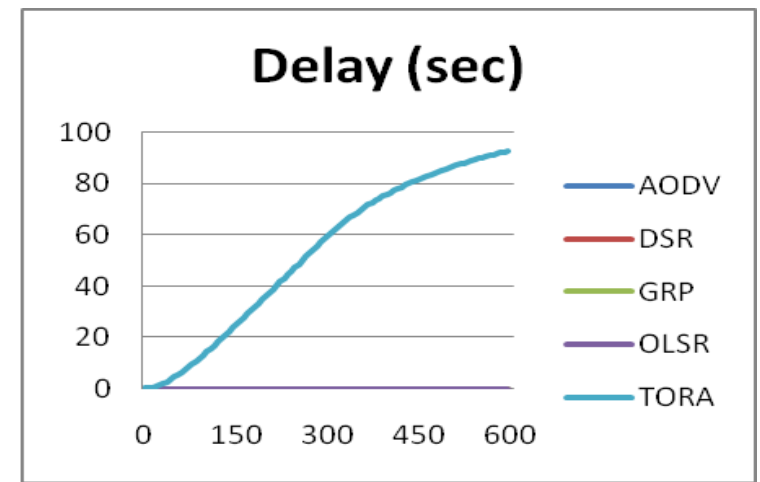

Fig. 3(a): Delay for 90 nodes for e-mail

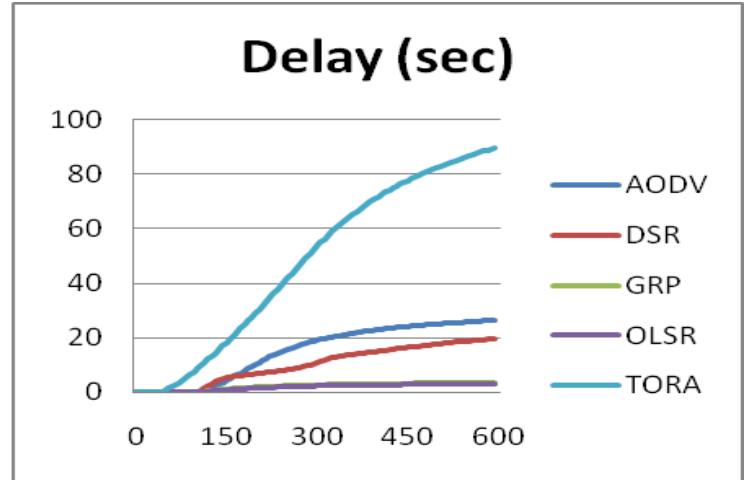

Fig. 3(b): Delay for 90 nodes for video conferencing

\subsection{Load}

The Fig. 4(a) shows the entire load for 30 nodes for email application. The DSR protocol shows least load followed by GRP and AODV. The TORA protocol shows highest load. The Fig. 4(b) shows the entire load for 30 nodes for video conferencing application. The AODV protocol shows least load followed by TORA and OLSR. The DSR protocol shows highest load.

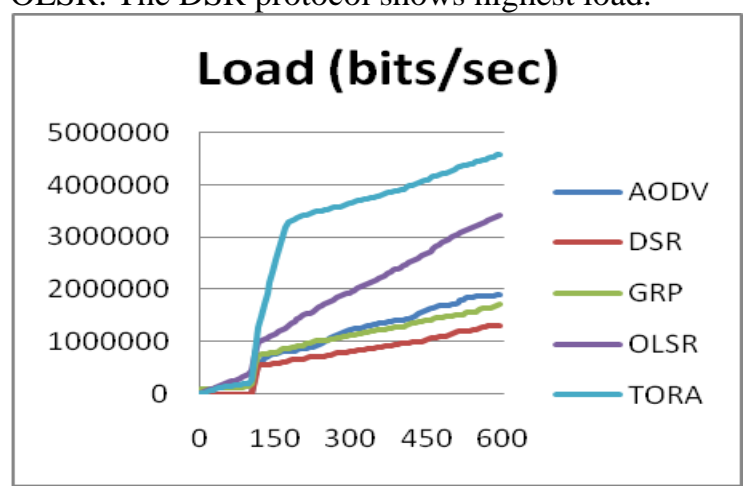

Fig. 4(a): Load for 30 nodes for e-mail

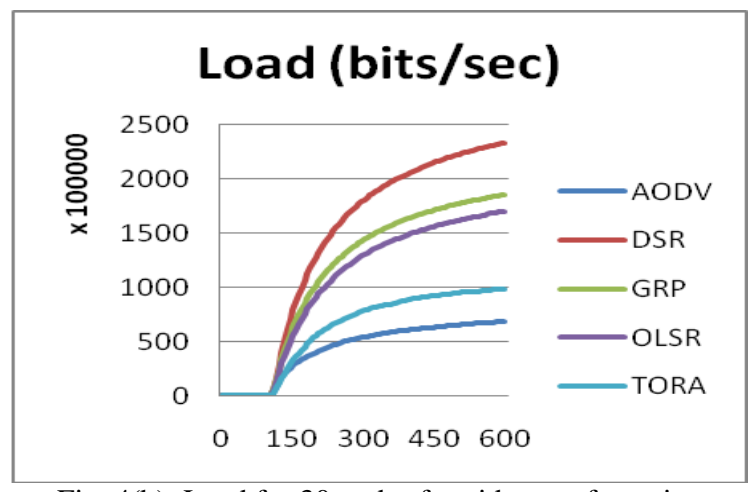

Fig. 4(b): Load for 30 nodes for video conferencing

The Fig. 5(a) shows the entire load for 60 nodes for email application. The DSR protocol shows least load followed by GRP and AODV. The TORA protocol shows highest load. The Fig. 5(b) shows the entire load for 60 nodes for video conferencing application. The TORA protocol shows least load followed by AODV and OLSR. The DSR protocol shows highest load. 


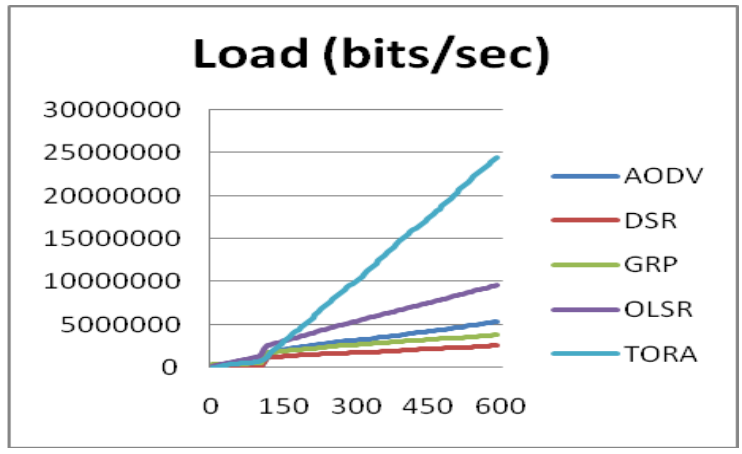

Fig. 5(a): Load for 60 nodes for e-mail

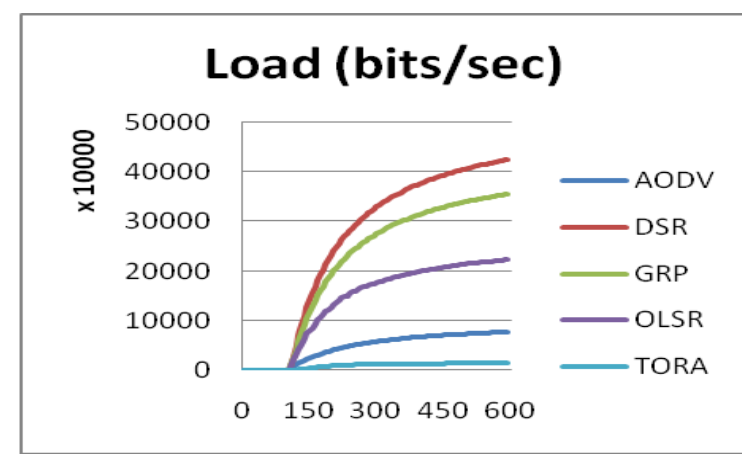

Fig. 5(b): Load for 60 nodes for video conferencing

The Fig. 6(a) shows the entire load for 90 nodes for email application. The DSR protocol shows least load followed by GRP and AODV. The TORA protocol shows highest load. The Fig. 6(b) shows the entire load for 90 nodes for video conferencing application. The TORA protocol shows least load followed by AODV and OLSR. The DSR protocol shows highest load.

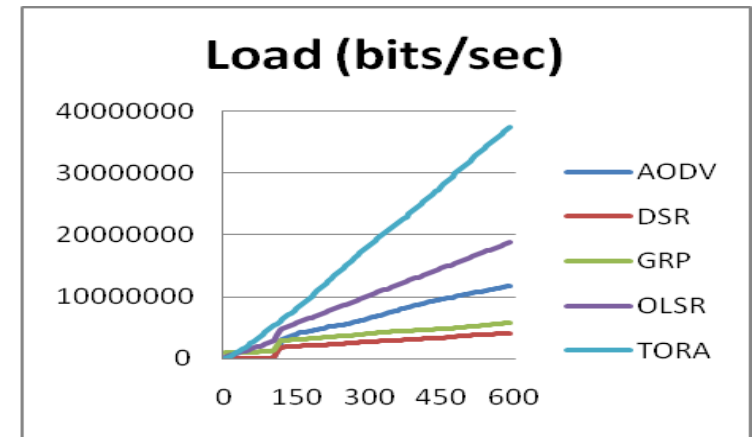

Fig. 6(a): Load for 90 nodes for e-mail

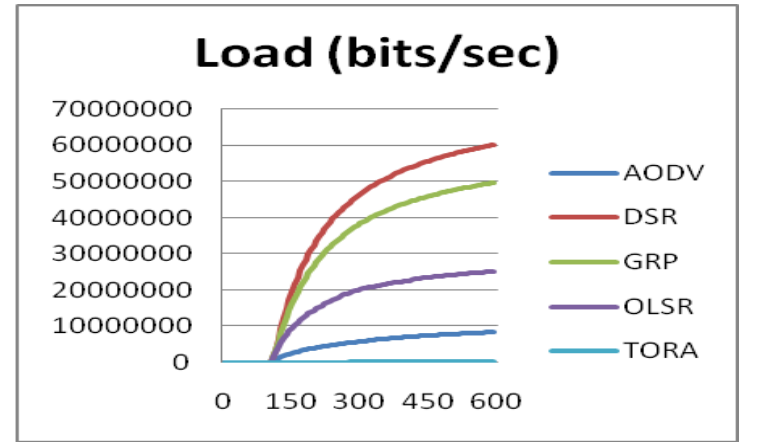

Fig. 6(b): Load for 90 nodes for video conferencing

\subsection{Throughput}

The Fig. 7(a) shows the throughput for 30 nodes for email. The OLSR protocol shows highest throughput which comes out to be 66782261 bits/sec followed by AODV. The DSR protocol shows least throughput. The Fig. 7(b) shows the entire throughput for 30 nodes for video conferencing application. The AODV protocol shows highest throughput which is 468088176 bits/sec followed by GRP. The TORA protocol shows least throughput.

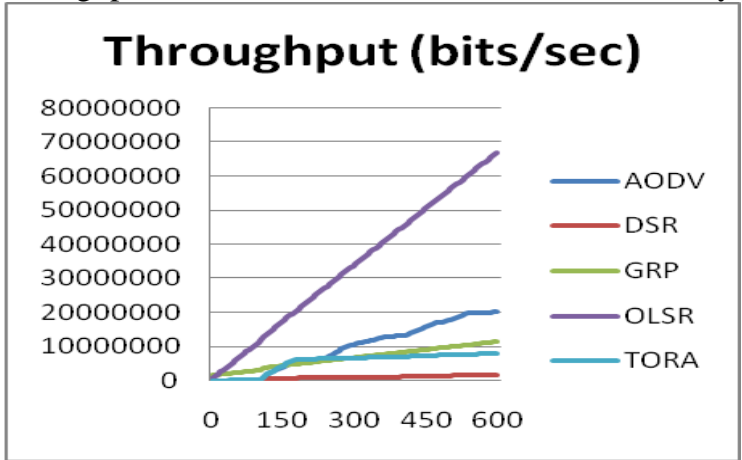

Fig. 7(a): Throughput for 30 nodes for e-mail
Throughput (bits/sec)

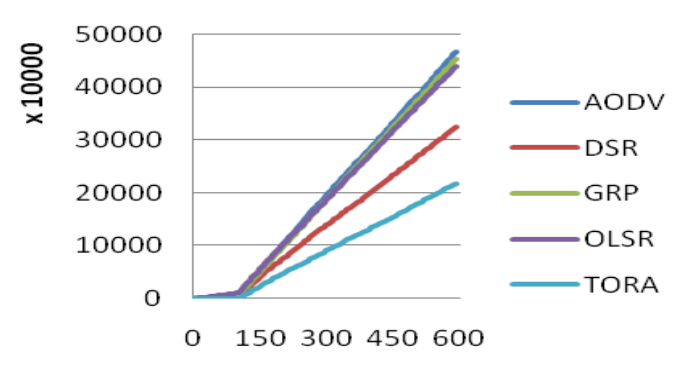

Fig.7 (b): Throughput for 30 nodes for video conferencing

The Fig. 8(a) shows the throughput for 60 nodes for email. The OLSR protocol shows highest throughput which comes out to be 435028495 bits/sec followed by AODV. The DSR protocol shows least throughput. The Fig. 8(b) shows the throughput for 60 nodes for video conferencing. The AODV protocol shows highest throughput which is 712636762 bits/sec followed by OLSR. The TORA protocol shows least throughput. 


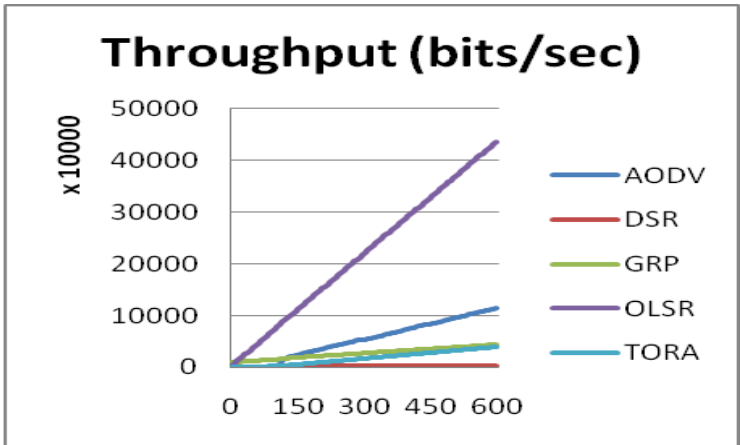

Fig. 8(a): Throughput for 60 nodes for e-mail

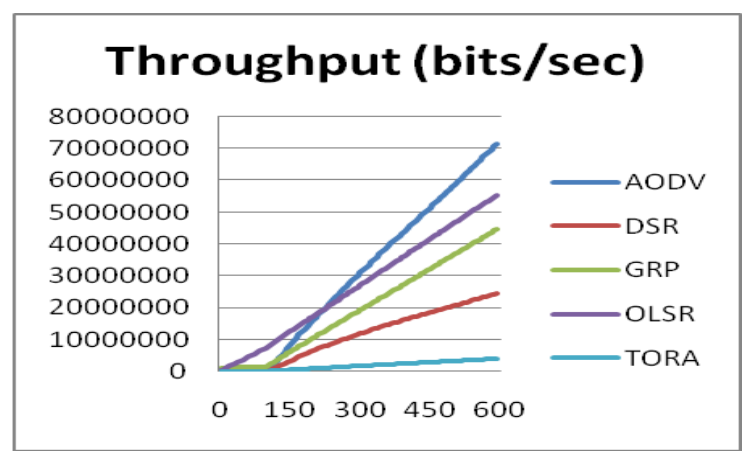

Fig.8(b):Throughput for 60 nodes for video conferencing The Fig. 9(a) shows the throughput for 90 nodes for email. The OLSR protocol shows highest throughput which comes out to be $1359717136 \mathrm{bits} / \mathrm{sec}$ followed by AODV. The DSR protocol shows least throughput. The Fig. 9(b) shows the throughput for 90 nodes for video conferencing. The AODV protocol shows highest throughput which is bits/sec followed by OLSR. The TORA protocol shows least throughput.

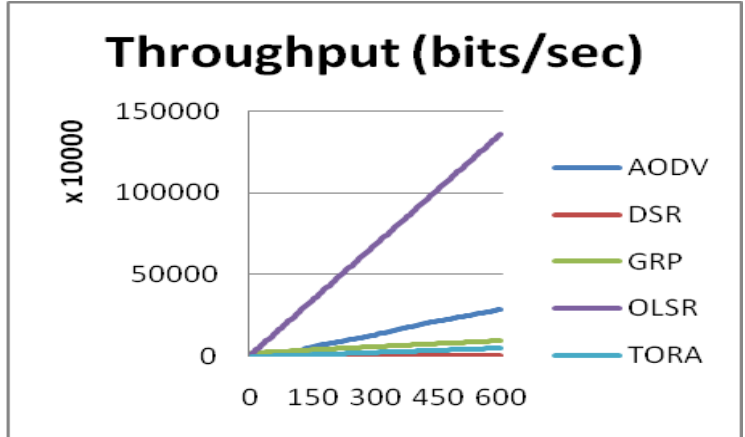

Fig. 9(a): Throughput for 90 nodes for e-mail

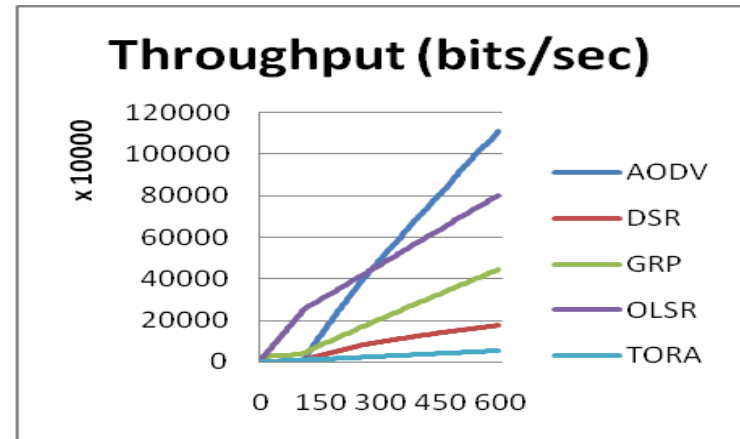

Fig.9(b):Throughput for 90 nodes for video conferencing

\subsection{Data Dropped}

The Fig. 10(a) shows the entire data drop for 30 nodes for email application. The TORA protocols shows data drop whereas rest all protocols show no drop. The Fig. 10(b) shows the entire data drop for 30 nodes for video conferencing application. The AODV protocol show least data drop followed by TORA. The GRP protocol shows highest data drop.

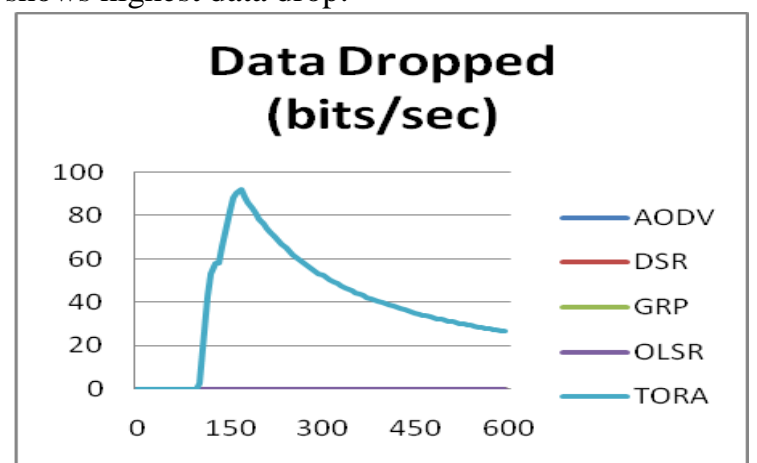

Fig. 10(a): Data Dropped for 30 nodes for e-mail

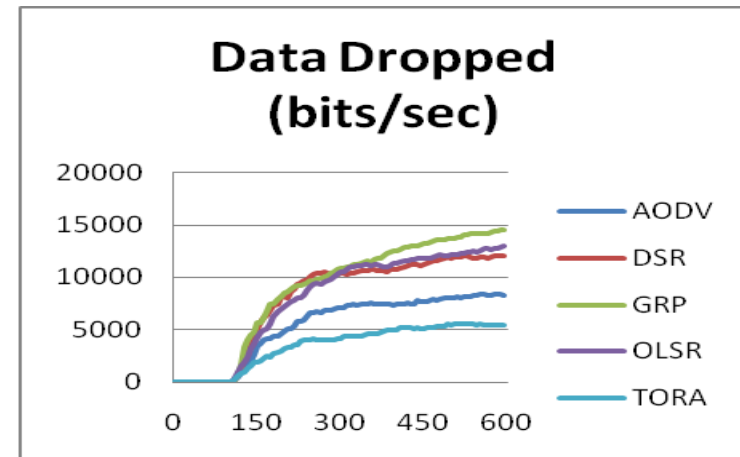

Fig. 10(b): Data Drop for 30 nodes for video conferencing The Fig. 11(a) shows the entire data drop for 60 nodes for email application. The TORA protocols shows data drop followed by AODV which shows almost negligible drop whereas rest all protocols show no drop. The Fig. 11(b) shows the entire data drop for 60 nodes for video conferencing application. The TORA protocol show least data drop followed by AODV. The GRP protocol shows highest data drop. 


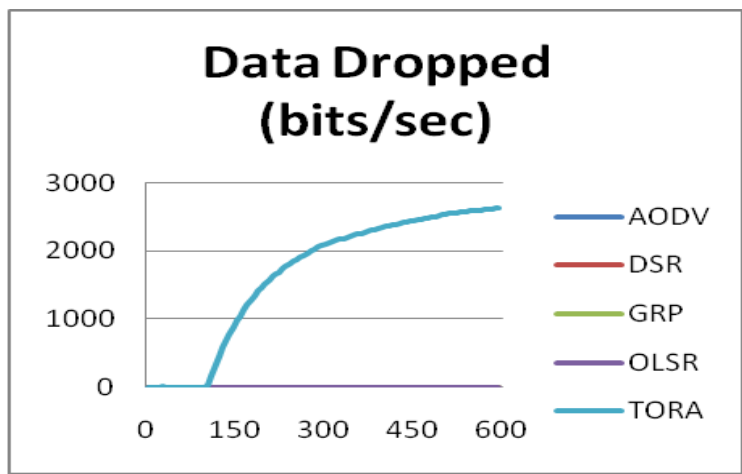

Fig. 11(a): Data Dropped for 60 nodes for e-mail

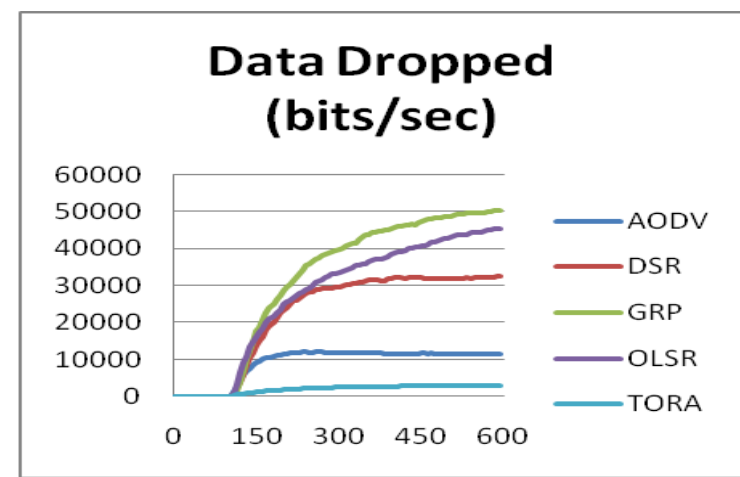

Fig. 11(b): Data Drop for 60 nodes for video conferencing The Fig. 12(a) shows the entire data drop for 90 nodes for email application. The AODV protocol shows least data drop followed by OLSR whereas the TORA protocol shows highest data drop. The Fig. 12(b) shows the entire data drop for 90 nodes for video conferencing application. The TORA protocol show least data drop followed by AODV. The GRP protocol shows highest data drop.

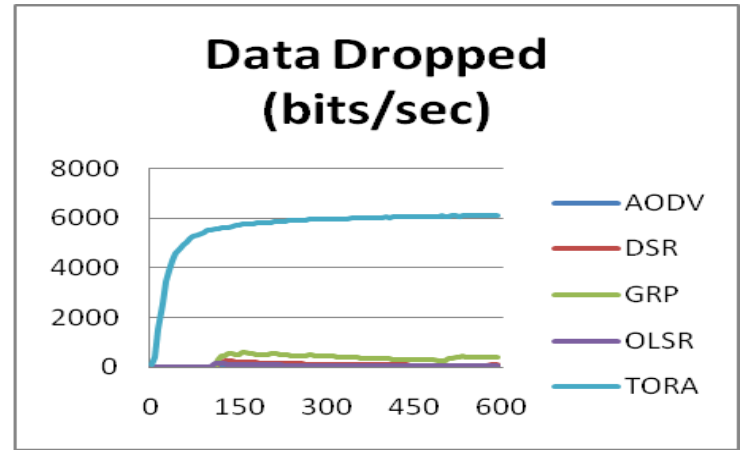

Fig. 10(a): Data Dropped for 90 nodes for e-mail

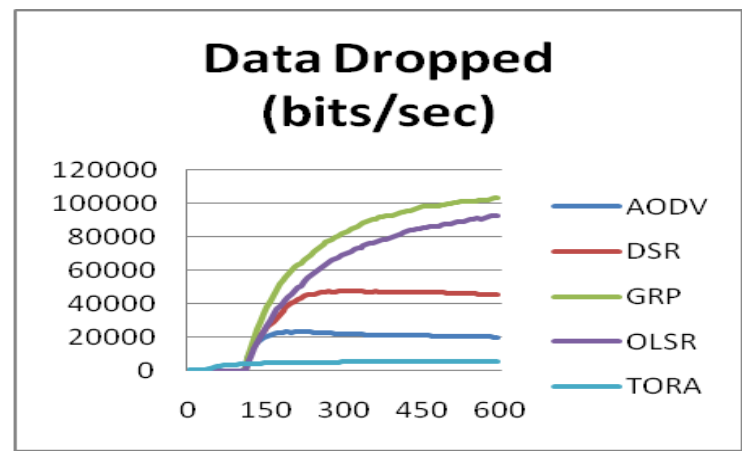

Fig. 10(b): Data Drop for 90 nodes for video conferencing

V.

\section{RESULT ANALYSIS}

This paper presented performance analysis of five routing protocols in MANET. We are already having number of routing protocols available so we can experiment on them and find out best suitable protocols for various scenarios as desired accordingly rather than developing new protocols.

First we discuss video conferencing with varying number of nodes. In this scenario following points are concluded:

- GRP has least delay but as the number of nodes is increased the delay for GRP increases but OLSR initially has high delay as compared to GRP but with increase in number of nodes the delay for OLSR decreases.

- Initially the load for AODV is low but it increases with increase in number of nodes but the load for TORA decreases with the increase in number of nodes.

- AODV shows highest throughput followed by OLSR and GRP protocol.

- The AODV protocol shops low data drop for 30 nodes but the data dropped increase with increase in number of nodes whereas the TORA protocol reduces the data drop with increase in number of nodes.

- TORA and DSR show poor performance with TORA exceptionally showing low data drop and load.

In the second scenario we consider e-mail as application for all number of nodes. In this scenario following points are concluded:

- GRP has least delay followed by OLSR protocol.

- DSR shows least load followed by GRP protocol.

- OLSR protocol shows highest throughput followed by AODV. GRP shows average value of throughput

- Initially all the protocols show no data drop for 30 and 60 nodes except for TORA which shows data drop. For 90 nodes the data dropped for GRP raises but AODV and OLSR shows low data dropped.

- TORA and DSR shows poor performance with DSR exceptionally showing low load and low data drop.

VI. CONCLUSION AND FUTURE WORK

The study of simulations with video conferencing application shows AODV has high throughput with low load and low data dropped but shows poor delay. GRP protocol shows low delay, good throughput but high 
data dropped and load. OLSR protocol shows poor performance for 30 nodes but its performance increases with increase in number of nodes. The AODV protocol is best suited protocol for 30 nodes and even for 60 nodes if we can tolerate delay. The GRP protocol is good choice if we can't tolerate delay. OLSR protocol is good choice for 90 nodes i.e. high number of nodes. Still AODV is best protocol for this scenario.

The study of simulations with email application shows that the OLSR has low delay, high throughput and a low data drop so the best protocol in this scenario. The GRP protocol shows low delay, low load but low throughput and data drop also increases.

The overall study of both the applications shows that AODV is best suited protocol for video conferencing for lower number of nodes and OLSR is can be used as a replacement as its performance degrades for high number of nodes. The OLSR protocol shows best performance for email traffic but GRP is also equally good. GRP performance is better for low number of nodes but it degrades with increase in number of nodes but OLSR improves its performance. TORA shows poor results in both scenarios followed by DSR. In both scenarios GRP and AODV suits the low number of nodes whereas OLSR suits better for high number of nodes.

In further continuance to this study we can analyze these protocols with varying area and loads and varying pause time and speed to further go into greater depth of the routing protocols.

\section{REFERENCES}

[1] Nagham H. Saeed, Maysam F. Abbod and Hamed S. Al-Raweshidy, "MANET Routing Protocol Taxonomy", Proc. of IEEE Conference on future Communication Networks (2012).

[2] G. Rajkumar, P. Kasiram and D. Parthiban, "Optimized QoS Metrics and performance comparation of DSR and AODV Routing Protocols", Proc. of IEEE Conference on advances in Engineering, Science and Management (ICAESM-2012), March 30-31, 2012.

[3] Avni Khatkar and Yudhvir Singh, "Performance Evaluation of Hybrid Routing Protocols in Mobile Adhoc Networks", Proc. of 2 ${ }^{\text {nd }}$ IEEE Conference on Advanced Computing \& Communication Technologies (ICACC-2012).

[4] Mehdi Barati, Kayvan Atefi, Farshad Khosravi and Yashar Azab Daftari, "Performance Evaluation of Energy Consumption for AODV and DSR Routing Protocols in MANET", Proc. of IEEE Conference on Computer \& Information Science (ICCIS-2012).

[5] Mina Vajed Khiavi, Shahram Jamali, Sajjad Jahanbakhsh Gudakahriz, "Performance comparison of AODV, DSDV, DSR, TORA routing protocols in MANETs", International Research Journal of Applied and Basic Sciences, Vol. 3(7), 2012.

[6] Parul Sharma, Arvind Kalia and Jawahar Thakur, "Performance analysis of AODV, DSR and DSDV Routing Protocols in Mobile Adhoc Networks (MANET)", Journal of Information Systems and Communication, Vol. 3, Issue 1, 2012.

[7] Manveen Singh Chadha, Ranbir Joon and Sandeep, "Simulation and Comparison of AODV, DSR and AOMDV Routing Protocols in MANETs", International Journal of Soft Computing and Engineering (IJSCE), Vol. 2, Issue 3, July 2012.

[8] Muhammad Shaffatul Islam, Md. Adnan Riaz, Mohammed Tarique, "Performance analysis of the routing protocols for video streaming over Mobile ad hoc networks", International Journal of Computer Networks \& Communications (IJCNC), Vol. 4, No. 3, May 2012.

[9] Laxmi Shrivastava, Sarita S. Bhadauria and G.S. Tomar, "Performance Evaluation of Routing Protocols in MANET with different traffic loads", Proc. of IEEE Conference on Communication Systems and Network Technologies(CSNT 2011).

[10] Pragati, Rajender Nath, "Performance evaluation of AODV, LEACH, TORA protocols through simulation", International journal of advanced research in computer science and software engineering, Vol. 2, Issue 7, July 2012.

[11] Punardeep Singh, Harpal Singh, Satnderpal Ahuja, "Brief description of routing protocols in MANETs and performance and analysis(AODV, AOMDV, TORA)", International Journal of Advanced research in computer Science and Software Engineering, Vol. 2, Issue 1, January 2012.

[12] P. Kuppusamy, K. Thirunavukkarsu and B. Kalavathi, "A study and Comparison of OLSR, AODV and TORA Routing Protocols in Ad hoc Networks", Proc. of $3^{\text {rd }}$ IEEE Conference on Electronics Computer Technology (ICECT 2011), 8-10 April 2011

[13] Fahim Maan and Nauman Mazhar, "MANET Routing Protocols vs Mobility Models: A Performance Evaluation", Proc. of IEEEICUFN 2011 